\title{
Unitarily invariant norm inequalities for some means
}

\section{Shigeru Furuichi*}

\section{"Correspondence:}

furuichi@chs.nihon-u.ac.jp

Department of Information Science,

College of Humanities and Sciences,

Nihon University, Sakurajyousui,

Setagaya-ku, Tokyo, 156-8550, Japan

\begin{abstract}
We introduce some symmetric homogeneous means, and then we show unitarily invariant norm inequalities for them, applying the method established by Hiai and Kosaki. Our new inequalities give tighter bounds of the logarithmic mean than the inequalities given by Hiai and Kosaki. Some properties and norm continuities in the parameter for our means are also discussed.
\end{abstract}

MSC: $15 \mathrm{~A} 39 ; 15 \mathrm{~A} 45$

Keywords: symmetric homogeneous mean; logarithmic mean; unitarily invariant norm and norm inequality

\section{Introduction}

In the previous paper, we derived tight bounds for the logarithmic mean in the case of the Frobenius norm, inspired by the work of Zou in [1].

Theorem 1.1 ([2]) For any matrices $S, T, X$ with $S, T \geq 0, m_{1} \geq 1, m_{2} \geq 2$ and Frobenius norm $\|\cdot\|_{F}$, the following inequalities hold:

$$
\begin{aligned}
\frac{1}{m_{1}}\left\|\sum_{k=1}^{m_{1}} S^{k /\left(m_{1}+1\right)} X T^{\left(m_{1}+1-k\right) /\left(m_{1}+1\right)}\right\|_{F} & \leq \frac{1}{m_{1}}\left\|\sum_{k=1}^{m_{1}} S^{(2 k-1) / 2 m_{1}} X T^{\left(2 m_{1}-(2 k-1)\right) / 2 m_{1}}\right\|_{F} \\
& \leq\left\|\int_{0}^{1} S^{\nu} X T^{1-v} d v\right\|_{F} \\
& \leq \frac{1}{m_{2}}\left\|\sum_{k=0}^{m_{2}} S^{k / m_{2}} X T^{\left(m_{2}-k\right) / m_{2}}-\frac{1}{2}(S X+X T)\right\|_{F} \\
& \leq \frac{1}{m_{2}}\left\|\sum_{k=0}^{m_{2}-1} S^{k /\left(m_{2}-1\right)} X T^{\left(m_{2}-1-k\right) /\left(m_{2}-1\right)}\right\|_{F}
\end{aligned}
$$

Although the Frobenius norm is only a special case of unitarily invariant norm, our bounds for the logarithmic mean have improved those in the following results by Hiai and Kosaki [3, 4].

@2014 Furuichi; licensee Springer. This is an Open Access article distributed under the terms of the Creative Commons Attribution License (http://creativecommons.org/licenses/by/2.0), which permits unrestricted use, distribution, and reproduction in any medium, provided the original work is properly cited. 
Theorem $1.2([3,4])$ For any bounded linear operators $S, T, X$ with $S, T \geq 0, m_{1} \geq 1, m_{2} \geq$ 2 and any unitarily invariant norm ||$\cdot \mid \|$, the following inequalities hold:

$$
\begin{aligned}
\left\|S^{1 / 2} X T^{1 / 2}\right\| & \leq \frac{1}{m_{1}}\left\|\sum_{k=1}^{m_{1}} S^{k /\left(m_{1}+1\right)} X T^{\left(m_{1}+1-k\right) /\left(m_{1}+1\right)}\right\| \leq\left\|\int_{0}^{1} S^{\nu} X T^{1-v} d v\right\| \| \\
& \leq \frac{1}{m_{2}}\left\|\sum_{k=0}^{m_{2}-1} S^{k /\left(m_{2}-1\right)} X T^{\left(m_{2}-1-k\right) /\left(m_{2}-1\right)}\right\| \leq \frac{1}{2}\|S X+X T\| .
\end{aligned}
$$

In this paper, we give tighter bounds for the logarithmic mean than those by Hiai and Kosaki $[3,4]$ for every unitarily invariant norm. That is, we give the generalized results of Theorem 1.1 for the unitarily invariant norm. For this purpose, we firstly introduce two quantities.

Definition 1.3 For $\alpha \in \mathbb{R}$ and $x, y>0$, we set

$$
P_{\alpha}(x, y) \equiv\left\{\begin{array} { l l } 
{ \frac { \alpha x ^ { \alpha } ( x - y ) } { x ^ { \alpha } - y ^ { \alpha } } } & { ( x \neq y ) , } \\
{ x } & { ( x = y ) , }
\end{array} \quad \text { and } \quad Q _ { \alpha } ( x , y ) \equiv \left\{\begin{array}{ll}
\frac{\alpha y^{\alpha}(x-y)}{x^{\alpha}-y^{\alpha}} & (x \neq y) \\
x & (x=y)
\end{array}\right.\right.
$$

We note that we have the following bounds of logarithmic mean with the above two means (see the appendix in the paper [2]):

$$
\begin{cases}Q_{1 / m}(x, y)<L M(x, y)<P_{1 / m}(x, y) & (\text { if } x>y) \\ P_{1 / m}(x, y)<L M(x, y)<Q_{1 / m}(x, y) & (\text { if } x<y)\end{cases}
$$

where the logarithmic mean is defined by

$$
\operatorname{LM}(x, y) \equiv \begin{cases}\frac{x-y}{\log x-\log y} & (x \neq y) \\ x & (x=y)\end{cases}
$$

We here define a few symmetric homogeneous means using $P_{\alpha}(x, y)$ and $Q_{\alpha}(x, y)$ in the following way.

\section{Definition 1.4}

(i) For $|\alpha| \leq 1$ and $x \neq y$, we define

$$
A_{\alpha}(x, y) \equiv \frac{1}{2} P_{\alpha}(x, y)+\frac{1}{2} Q_{\alpha}(x, y)=\frac{\alpha\left(x^{\alpha}+y^{\alpha}\right)(x-y)}{2\left(x^{\alpha}-y^{\alpha}\right)} .
$$

(ii) For $\alpha \in \mathbb{R}$ and $x \neq y$, we define

$$
L_{\alpha}(x, y) \equiv \frac{P_{\alpha}(x, y)-Q_{\alpha}(x, y)}{\log P_{\alpha}(x, y)-\log Q_{\alpha}(x, y)}=L M(x, y)
$$

which is independent of $\alpha$.

(iii) For $|\alpha| \leq 2$ and $x \neq y$, we define

$$
G_{\alpha}(x, y) \equiv \sqrt{P_{\alpha}(x, y) Q_{\alpha}(x, y)}=\frac{\alpha(x y)^{\alpha / 2}(x-y)}{x^{\alpha}-y^{\alpha}}
$$


(iv) For $|\alpha| \leq 1$ and $x \neq y$, we define

$$
H_{\alpha}(x, y) \equiv \frac{2 P_{\alpha}(x, y) Q_{\alpha}(x, y)}{P_{\alpha}(x, y)+Q_{\alpha}(x, y)}=\frac{2 \alpha(x y)^{\alpha}}{x^{\alpha}+y^{\alpha}} \frac{(x-y)}{x^{\alpha}-y^{\alpha}}
$$

and we also set $A_{\alpha}(x, y)=L_{\alpha}(x, y)=G_{\alpha}(x, y)=H_{\alpha}(x, y)=x$ for $x=y$.

We have the following relations for the above means:

$$
\begin{aligned}
& A_{1}(x, y)=A M(x, y) \equiv \frac{1}{2}(x+y), \quad A_{0}(x, y)=\lim _{\alpha \rightarrow 0} A_{\alpha}(x, y)=L M(x, y) \\
& G_{0}(x, y)=\lim _{\alpha \rightarrow 0} G_{\alpha}(x, y)=L M(x, y), \quad G_{1}(x, y)=G M(x, y) \equiv \sqrt{x y} \\
& G_{2}(x, y)=H M(x, y) \equiv \frac{2 x y}{x+y}, \quad H_{0}(x, y)=\lim _{\alpha \rightarrow 0} H_{\alpha}(x, y)=L M(x, y) \\
& H_{1 / 2}(x, y)=G M(x, y), \quad H_{1}(x, y)=H M(x, y)
\end{aligned}
$$

and $H_{\alpha}(x, y)=G_{2 \alpha}(x, y)$. In addition, the above means are written as the following geometric bridges:

$$
\begin{aligned}
& A_{\alpha}(x, y)=\left[B_{\alpha}(x, y)\right]^{\alpha}\left[S_{\alpha}(x, y)\right]^{1-\alpha}, \quad L_{\alpha}(x, y)=\left[E_{\alpha}(x, y)\right]^{\alpha}\left[S_{\alpha}(x, y)\right]^{1-\alpha}, \\
& G_{\alpha}(x, y)=[G M(x, y)]^{\alpha}\left[S_{\alpha}(x, y)\right]^{1-\alpha}, \quad H_{\alpha}(x, y)=\left[D_{\alpha}(x, y)\right]^{\alpha}\left[S_{\alpha}(x, y)\right]^{1-\alpha},
\end{aligned}
$$

where

$$
S_{\alpha}(x, y) \equiv\left(\frac{\alpha(x-y)}{x^{\alpha}-y^{\alpha}}\right)^{1 /(1-\alpha)}, \quad B_{\alpha}(x, y) \equiv\left(\frac{x^{\alpha}+y^{\alpha}}{2}\right)^{1 / \alpha}
$$

and

$$
D_{\alpha}(x, y) \equiv\left(\frac{2 x^{\alpha} y^{\alpha}}{x^{\alpha}+y^{\alpha}}\right)^{1 / \alpha}, \quad E_{\alpha}(x, y) \equiv\left(\frac{x^{\alpha}-y^{\alpha}}{\alpha(\log x-\log y)}\right)^{1 / \alpha}
$$

$S_{\alpha}(x, y)$ and $B_{\alpha}(x, y)$ are called Stolarsky mean and binomial mean, respectively.

In the previous paper [2], as tight bounds of logarithmic mean, the scalar inequalities were shown

$$
G_{1 / m}(x, y) \leq L M(x, y) \quad(m \geq 1), \quad L M(x, y) \leq A_{1 / m}(x, y) \quad(m \geq 2)
$$

which equivalently implied Frobenius norm inequalities (Theorem 1.1). See Theorem 2.2 and Theorem 3.2 in [2] for details. In this paper, we give unitarily invariant norm inequalities which are general results including Frobenius norm inequalities as a special case.

\section{Unitarily invariant norm inequalities}

To obtain unitarily invariant norm inequalities, we apply the method established by Hiai and Kosaki [4-7].

Definition 2.1 A continuous positive real function $M(x, y)$ for $x, y>0$ is called a symmetric homogeneous mean if the function $M$ satisfies the following properties: 
(a) $M(x, y)=M(y, x)$.

(b) $M(c x, c y)=c M(x, y)$ for $c>0$.

(c) $M(x, y)$ is non-decreasing in $x, y$.

(d) $\min \{x, y\} \leq M(x, y) \leq \max \{x, y\}$.

The functions $A_{\alpha}(x, y), L_{\alpha}(x, y), G_{\alpha}(x, y), H_{\alpha}(x, y)$ defined in Definition 1.4 are symmetric homogeneous means. We give powerful theorem to obtain unitarily invariant norm inequalities. In the references [4-7], another equivalent conditions were given. However, here we give minimum conditions to obtain our results in this paper. Throughout this paper, we use the symbol $B(\mathcal{H})$ as the set of all bounded linear operators on a separable Hilbert space $\mathcal{H}$. We also use the notation $K \geq 0$ if $K \in B(\mathcal{H})$ satisfies $\langle K x, x\rangle \geq 0$ for all $x \in \mathcal{H}$ (then $K$ is called a positive operator).

Theorem 2.2 ([4-7]) For two symmetric homogeneous means $M$ and $N$, the following conditions are equivalent:

(i) \|\|$M(S, T) X\|\leq\| N(S, T) X \|$ for any $S, T, X \in B(\mathcal{H})$ with $S, T \geq 0$ and for any unitarily invariant norm $\|\cdot \cdot\| \mid$.

(ii) The function $M\left(e^{t}, 1\right) / N\left(e^{t}, 1\right)$ is positive definite function on $\mathbb{R}$ (then we denote $M \preceq N$ ), where the positive definiteness of a real continuous function $\phi$ on $\mathbb{R}$ means that $\left[\phi\left(t_{i}-t_{j}\right)\right]_{i, j=1, \ldots, n}$ is positive definite for any $t_{1}, \ldots, t_{n} \in \mathbb{R}$ with any $n \in \mathbb{N}$.

Thanks to Theorem 2.2, our task to obtain unitarily invariant norm inequalities in this paper is to show the relation $M \preceq N$, which is stronger than the usual scalar inequalities $M \leq N$. That is, $M(s, t) \preceq N(s, t)$ implies $M(s, t) \leq N(s, t)$.

We firstly give monotonicity of three means $H_{\alpha}(x, y), G_{\alpha}(x, y)$ and $A_{\alpha}(x, y)$ for the parameter $\alpha \in \mathbb{R}$. Since we have $H_{-\alpha}(x, y)=H_{\alpha}(x, y), G_{-\alpha}(x, y)=G_{\alpha}(x, y)$ and $A_{-\alpha}(x, y)=A_{\alpha}(x, y)$, we consider the case $\alpha \geq 0$. Then we have the following proposition.

\section{Proposition 2.3}

(i) If $0 \leq \alpha<\beta \leq 1$, then $H_{\beta} \preceq H_{\alpha}$.

(ii) If $0 \leq \alpha<\beta \leq 2$, then $G_{\beta} \preceq G_{\alpha}$.

(iii) If $0 \leq \alpha<\beta \leq 1$, then $A_{\alpha} \preceq A_{\beta}$.

Proof

(i) We calculate

$$
\frac{H_{\beta}\left(e^{t}, 1\right)}{H_{\alpha}\left(e^{t}, 1\right)}=\frac{2 \beta e^{\beta t}\left(e^{t}-1\right)}{e^{2 \beta t}-1} \cdot \frac{e^{2 \alpha t}-1}{2 \alpha e^{\alpha t}\left(e^{t}-1\right)}=\frac{\beta}{\alpha} \cdot \frac{e^{\beta t}\left(e^{2 \alpha t}-1\right)}{e^{\alpha t}\left(e^{2 \beta t}-1\right)}=\frac{\beta}{\alpha} \frac{\sinh \alpha t}{\sinh \beta t} .
$$

This is a positive definite function for the case $\alpha<\beta$, so that we have $H_{\beta} \preceq H_{\alpha}$.

(ii) The similar calculation

$$
\frac{G_{\beta}\left(e^{2 t}, 1\right)}{G_{\alpha}\left(e^{2 t}, 1\right)}=\frac{2 \beta e^{\beta t}\left(e^{2 t}-1\right)}{e^{2 \beta t}-1} \cdot \frac{e^{2 \alpha t}-1}{2 \alpha e^{\alpha t}\left(e^{2 t}-1\right)}=\frac{\beta}{\alpha} \cdot \frac{e^{\beta t}\left(e^{2 \alpha t}-1\right)}{e^{\alpha t}\left(e^{2 \beta t}-1\right)}=\frac{\beta}{\alpha} \cdot \frac{\sinh \alpha t}{\sinh \beta t}
$$

implies $G_{\beta} \preceq G_{\alpha}$. 
(iii) Since the case $0=\alpha<\beta \leq 1$ follows from the limit of the case $0<\alpha<\beta \leq 1$, we may assume $0<\alpha<\beta \leq 1$. Since we have

$$
\frac{A_{\alpha}\left(e^{2 t}, 1\right)}{A_{\beta}\left(e^{2 t}, 1\right)}=\frac{\alpha}{\beta} \cdot \frac{\sinh \beta t \cosh \alpha t}{\cosh \beta t \sinh \alpha t}
$$

we calculate by the formula $\sinh (x)=2 \cosh (x / 2) \sinh (x / 2)$ repeatedly

$$
\begin{aligned}
\frac{\sinh \beta t \cosh \alpha t}{\cosh \beta t \sinh \alpha t}-1 & =\frac{\sinh (\beta-\alpha) t}{\cosh \beta t \sinh \alpha t}=\frac{2 \cosh \left(\frac{\beta-\alpha}{2} t\right) \sinh \left(\frac{\beta-\alpha}{2} t\right)}{\cosh \beta t \sinh \alpha t} \\
& =\lim _{n \rightarrow \infty} \frac{2^{n} \prod_{k=1}^{n} \cos \left(\frac{\beta-\alpha}{2^{k}} t\right) \sinh \left(\frac{\beta-\alpha}{2^{n}} t\right)}{\cosh \beta t \sinh \alpha t} .
\end{aligned}
$$

From Proposition 4 in [8], the sufficient condition that the function $\left(\prod_{k=1}^{n} \cosh \left(\left((\beta-\alpha) / 2^{k}\right) t\right)\right) / \cosh \beta t$ is positive definite is $\sum_{k=1}^{n}\left((\beta-\alpha) / \beta 2^{k}\right) \leq 1$, i.e., $(\beta-\alpha)\left(1-2^{-n}\right) \leq \beta$. The sufficient condition that the function $\left(\sinh \left(\left((\beta-\alpha) / 2^{n}\right) t\right)\right) / \sinh \alpha t$ is positive definite is $(\beta-\alpha) / 2^{n} \leq \alpha$. The conditions $(\beta-\alpha)\left(1-2^{-n}\right) \leq \beta$ and $(\beta-\alpha) / 2^{n} \leq \alpha$ are satisfied with a natural number $n$ sufficiently large. Thus we conclude $A_{\alpha} \preceq A_{\beta}$.

It may be notable that (iii) of the above proposition can be proven by a similar argument to Theorem 2.1 of the paper [4].

Next we give the relation among the four means $H_{\alpha}(x, y), G_{\alpha}(x, y), L_{\alpha}(x, y)$, and $A_{\alpha}(x, y)$.

Proposition 2.4 For any $S, T, X \in B(\mathcal{H})$ with $S, T \geq 0,|\alpha| \leq 1$ and any unitarily invariant norm $\|\mid \cdot\|$, we have

$$
\left\|H_{\alpha}(S, T) X\right\| \leq\left\|G_{\alpha}(S, T) X\right\| \leq\left\|L_{\alpha}(S, T) X\right\| \leq\left\|A_{\alpha}(S, T) X\right\| .
$$

Proof We firstly calculate

$$
\frac{H_{\alpha}\left(e^{t}, 1\right)}{G_{\alpha}\left(e^{t}, 1\right)}=\frac{2 \alpha e^{\alpha t}}{e^{\alpha t}+1} \frac{\left(e^{t}-1\right)}{e^{\alpha t}-1} \frac{e^{\alpha t}-1}{\alpha e^{\alpha t / 2}\left(e^{t}-1\right)}=\frac{2 e^{\alpha t / 2}}{e^{\alpha t}+1}=\frac{2}{e^{\alpha t / 2}+e^{-\alpha t / 2}}=\frac{1}{\cosh \frac{\alpha t}{2}},
$$

which is a positive definite function. Thus we have $H_{\alpha} \preceq G_{\alpha}$ so that the first inequality of this proposition holds thanks to Theorem 2.2.

The calculation

$$
\frac{G_{\alpha}\left(e^{t}, 1\right)}{L_{\alpha}\left(e^{t}, 1\right)}=\frac{\alpha e^{\alpha t / 2}\left(e^{t}-1\right)}{e^{\alpha t}-1} \cdot \frac{t}{e^{t}-1}=\frac{\alpha t}{e^{\alpha t / 2}-e^{-\alpha t / 2}}=\frac{\frac{\alpha t}{2}}{\sinh \frac{\alpha t}{2}}
$$

implies $G_{\alpha} \preceq L_{\alpha}$. Thus we have the second inequality of this proposition.

Finally the calculation

$$
\frac{L_{\alpha}\left(e^{t}, 1\right)}{A_{\alpha}\left(e^{t}, 1\right)}=\frac{e^{t}-1}{t} \cdot \frac{2\left(e^{\alpha t}-1\right)}{\alpha\left(e^{\alpha t}+1\right)\left(e^{t}-1\right)}=\frac{2}{\alpha t} \cdot \frac{e^{\alpha t / 2}-e^{-\alpha t / 2}}{e^{\alpha t / 2}+e^{-\alpha t / 2}}=\frac{\tanh \frac{\alpha t}{2}}{\frac{\alpha t}{2}}
$$

implies $L_{\alpha} \preceq A_{\alpha}$. Thus we have the third inequality of this proposition. 
In the papers $[3,4]$, the unitarily invariant norm inequalities of the power difference mean (or A-L-G interpolating mean) $M_{\alpha}(x, y)$ was systematically studied. We give the relation for our means with the power difference mean:

$$
M_{\alpha}(x, y) \equiv \begin{cases}\frac{\alpha-1}{\alpha} \cdot \frac{x^{\alpha}-y^{\alpha}}{x^{\alpha-1}-y^{\alpha-1}} & (x \neq y), \\ x & (x=y) .\end{cases}
$$

Theorem 2.5 For any $S, T, X \in B(\mathcal{H})$ with $S, T \geq 0, m \in \mathbb{N}$ and any unitarily invariant norm $\||\cdot|\|$, we have

$$
\begin{aligned}
\left\|M_{m /(m+1)}(S, T) X\right\| & \leq\left\|G_{1 / m}(S, T) X\right\| \leq\|L(S, T) X\| \\
& \leq\left\|A_{1 / m}(S, T) X\right\| \leq\left\|M_{(m+1) / m}(S, T) X\right\| .
\end{aligned}
$$

(More concrete expressions of these inequalities will be written down in (8) of Section 4.)

Proof The second inequality and the third inequality have already been proven in Proposition 2.4 .

To prove the first inequality, for $0<\alpha, \beta<1$ we calculate

$$
\begin{aligned}
\frac{M_{\beta}\left(e^{2 t}, 1\right)}{G_{\alpha}\left(e^{2 t}, 1\right)} & =\frac{\beta-1}{\beta} \cdot \frac{e^{2 \beta t}-1}{e^{2(\beta-1) t}-1} \cdot \frac{e^{2 \alpha t}-1}{\alpha e^{\alpha t}\left(e^{2 t}-1\right)}=\frac{1-\beta}{\alpha \beta} \cdot \frac{\sinh \beta t}{\sinh t} \cdot \frac{\sinh \alpha t}{\sinh (1-\beta) t} \\
& =\frac{2(1-\beta)}{\alpha \beta} \cdot \frac{\sinh \beta t \cosh \frac{\alpha t}{2}}{\sinh t} \cdot \frac{\sinh \frac{\alpha t}{2}}{\sinh (1-\beta) t} .
\end{aligned}
$$

By Proposition 5 in [8], the function $(\sinh \beta t \cosh \alpha t / 2) / \sinh t$ is positive definite, if $\beta+$ $\alpha / 2 \leq 1$ and $\alpha / 2 \leq \frac{1}{2}$. The function $(\sinh \alpha t / 2) / \sinh (1-\beta) t$ is also positive definite, if $\alpha / 2 \leq$ $1-\beta$. The case $\alpha=1 / m$ and $\beta=m /(m+1)$ satisfies the above conditions. Thus we have $M_{m /(m+1)} \preceq G_{1 / m}$ which leads to the first inequality of this proposition.

To prove the last inequality, for $0<\alpha<1$ and $\beta>1$, we also calculate

$$
\begin{aligned}
\frac{A_{\alpha}\left(e^{2 t}, 1\right)}{M_{\beta}\left(e^{2 t}, 1\right)} & =\frac{\alpha \beta}{2(\beta-1)} \cdot \frac{\sinh t \sinh (\beta-1) t}{\tanh \alpha t \sinh \beta t}=\frac{\alpha \beta}{2(\beta-1)} \cdot \frac{\sinh t \cosh \alpha t \sinh (\beta-1) t}{\sinh \beta t \sinh \alpha t} \\
& =\frac{\alpha \beta}{2(\beta-1)} \cdot \frac{\sinh \frac{1}{\beta}(\beta t) \cosh \frac{\alpha}{\beta}(\beta t)}{\sinh \beta t} \cdot \frac{\sinh (\beta-1) t}{\sinh \alpha t} .
\end{aligned}
$$

By Proposition 5 in [8], the function $(\sinh 1 / \beta(\beta t) \cosh \alpha / \beta(\beta t)) / \sinh \beta t$ is positive definite, if $1 / \beta+\alpha / \beta \leq 1$ and $\alpha / \beta \leq \frac{1}{2}$. The function $(\sinh (\beta-1) t) / \sinh \alpha t$ is also positive definite, if $\beta-1 \leq \alpha$. From these conditions, we have $\beta=\alpha+1$ and $\alpha \leq 1$. The case $\alpha=1 / \mathrm{m}$ and $\beta=(m+1) / m$ satisfies the above conditions. Thus we have $A_{1 / m} \preceq M_{(m+1) / m}$, which leads to the last inequality.

Remark 2.6 Since $(m+1) / m<m /(m-1)$, by Theorem 2.1 in [4], we have $M_{(m+1) / m} \preceq$ $M_{m /(m-1)}$. Thus we have

$$
\left\|M_{(m+1) / m}(S, T) X\right\| \leq\left\|M_{m /(m-1)}(S, T) X\right\|,
$$

which means that Theorem 2.5 gives a general result for Theorem 1.1. 
Remark 2.7 From the well-known fact $M_{\alpha} \preceq M_{\beta}$ for $\alpha<\beta$, we have $H_{1}=H M=M_{-1} \preceq$ $M_{1 / 2}=G M$ and $H_{1 / 2}=G M=M_{1 / 2} \preceq M_{2 / 3}$. Thus we have

$$
\left\|H_{1 / m}(S, T) X\right\| \leq\left\|M_{m /(m+1)}(S, T) X\right\|
$$

for any $S, T, X \in B(\mathcal{H})$ with $S, T \geq 0, m=1,2$ and any unitarily invariant norm $\|\cdot\| \cdot \|$.

However, we do not have the scalar inequality $H_{1 / 3}(t, 1) \leq M_{3 / 4}(t, 1)$ for $t>0$ in general, so that the inequality (2) is not true for $m=3$. We also do not have the scalar inequality $H_{1 / 3}(t, 1) \geq M_{3 / 4}(t, 1)$ for $t>0$, in general.

\section{Norm continuity in parameter}

In this section, we consider the norm continuity argument with respect to the parameter on our introduced means. Since we have the relation $H_{\alpha}(x, y)=G_{2 \alpha}(x, y)$, we firstly consider the norm continuity in the parameter on $G_{\alpha}(S, T)$.

Proposition 3.1 Let $S, T, X \in B(\mathcal{H})$ with $S, T \geq 0$. If $0 \leq \alpha<\beta \leq 2$ and $\left\|G_{\alpha}(S, T) X\right\|<\infty$, then we have, for any unitarily invariant norm $\|\cdot\|$,

$$
\lim _{\beta^{\prime} \rightarrow \beta}\left\|G_{\beta}(S, T) X-G_{\beta^{\prime}}(S, T) X\right\|=0 .
$$

Proof From the following equality (see Eq. (1.4) in [4] for example):

$$
\frac{G_{\beta}\left(e^{2 t}, 1\right)}{G_{\alpha}\left(e^{2 t}, 1\right)}=\frac{\beta}{\alpha} \cdot \frac{\sinh \alpha t}{\sinh \beta t}=\frac{\beta}{\alpha} \int_{-\infty}^{\infty} e^{i t s} \frac{\sin \left(\frac{\pi \alpha}{\beta}\right)}{2 \beta\left\{\cosh \left(\frac{\pi s}{\beta}\right)+\cos \left(\frac{\pi \alpha}{\beta}\right)\right\}} d s,
$$

we have, for $0 \leq \alpha<\beta \leq 2$,

$$
G_{\beta}(S, T) X=\int_{-\infty}^{\infty}\left(S_{\text {supp } S}\right)^{i x}\left(G_{\alpha}(S, T) X\right)\left(T_{\text {supp } T}\right)^{-i x} \frac{\sin \left(\frac{\pi \alpha}{\beta}\right)}{2 \alpha\left\{\cosh \left(\frac{\pi S}{\beta}\right)+\cos \left(\frac{\pi \alpha}{\beta}\right)\right\}} d x
$$

applying Theorem 3.4 in [5] with $G_{\beta}(1,0)=0$. Here $S_{\text {supp } S}$ represents the support projection of $S$. Thus we have

$$
\begin{aligned}
\left\|G_{\beta}(S, T) X-G_{\beta^{\prime}}(S, T) X\right\| \leq & \left\|\frac{\sin \left(\frac{\pi \alpha}{\beta}\right)}{2 \alpha\left\{\cosh \left(\frac{\pi s}{\beta}\right)+\cos \left(\frac{\pi \alpha}{\beta}\right)\right\}}-\frac{\sin \left(\frac{\pi \alpha}{\beta^{\prime}}\right)}{2 \alpha\left\{\cosh \left(\frac{\pi s}{\beta^{\prime}}\right)+\cos \left(\frac{\pi \alpha}{\beta^{\prime}}\right)\right\}}\right\|_{1} \\
& \times\left\|G_{\alpha}(S, T) X\right\| \rightarrow 0 \quad\left(\beta \rightarrow \beta^{\prime}\right),
\end{aligned}
$$

by the Lebesgue dominated convergence theorem.

We secondly consider the norm continuity in the parameter on $A_{\alpha}(S, T)$.

Proposition 3.2 Let $S, T, X \in B(\mathcal{H})$ with $S, T \geq 0$. If $0<\alpha<\beta \leq 1$, then we have, for any unitarily invariant norm $\|\cdot\| \|$,

$$
\left\|A_{\alpha}(S, T) X\right\| \leq\left\|A_{\beta}(S, T) X\right\| \leq \frac{2 \beta-\alpha}{\alpha}\left\|A_{\alpha}(S, T) X\right\|
$$


and

$$
\left\|A_{\alpha}(S, T) X-A_{\beta}(S, T) X\right\| \leq \frac{2(\beta-\alpha)}{\alpha}\left\|A_{\alpha}(S, T) X\right\| .
$$

Proof The first inequality of (3) has been proved in (iii) of Proposition 2.3. Since $1 / \cosh \alpha t$ and $(\sinh (\beta-\alpha) t) / \sinh \beta t$ are positive definite functions,

$$
1-\frac{\alpha}{\beta} \cdot \frac{A_{\beta}\left(e^{2 t}, 1\right)}{A_{\alpha}\left(e^{2 t}, 1\right)}=\frac{\cosh \alpha t \sinh \beta t-\cosh \beta t \sinh \alpha t}{\cosh \alpha t \sinh \beta t}=\frac{1}{\cosh \alpha t} \cdot \frac{\sinh (\beta-\alpha) t}{\sinh \beta t}
$$

is positive definite. If we set

$$
A(s, t) \equiv \frac{\beta}{\beta-\alpha} A_{\alpha}(s, t)-\frac{\alpha}{\beta-\alpha} A_{\beta}(s, t),
$$

then we have $A\left(e^{t}, 1\right) / A_{\alpha}\left(e^{t}, 1\right)=\beta /(\beta-\alpha) \cdot 1 /(\cosh (\alpha t / 2)) \cdot((\sinh ((\beta-\alpha) t / 2)) /(\sinh (\beta t / 2)))$, which is a positive definite function. Thus we have

$$
\left\|\frac{\beta}{\beta-\alpha} A_{\alpha}(S, T) X-\frac{\alpha}{\beta-\alpha} A_{\beta}(S, T) X\right\| \leq\left\|A_{\alpha}(S, T) X\right\|
$$

by Theorem 2.4 in [7]. (Actually, $A(s, t)$ may not be a symmetric homogeneous mean. However, we easily find that it satisfies $A(s, t)=A(t, s)$ and $A(s, s)=s$. Then Theorem 2.4 in [7] ensures that the inequality (5) is valid.) Therefore we have

$$
\begin{aligned}
\frac{\alpha}{\beta-\alpha}\left\|A_{\beta}(S, T) X\right\| & \leq \frac{\beta}{\beta-\alpha}\left\|A_{\alpha}(S, T) X\right\|+\left\|\frac{\beta}{\beta-\alpha} A_{\alpha}(S, T) X-\frac{\alpha}{\beta-\alpha} A_{\beta}(S, T) X\right\| \| \\
& \leq\left(\frac{\beta}{\beta-\alpha}+1\right)\left\|A_{\alpha}(S, T) X\right\|=\frac{2 \beta-\alpha}{\beta-\alpha}\left\|A_{\alpha}(S, T) X\right\| \|,
\end{aligned}
$$

which is the second inequality of (3).

We prove the inequality (4):

$$
\begin{aligned}
& \left\|A_{\alpha}(S, T) X-A_{\beta}(S, T) X\right\| \\
& \quad=\left\|\left(1-\frac{\beta}{\alpha}\right) A_{\alpha}(S, T) X+\frac{\beta}{\alpha} A_{\alpha}(S, T) X-A_{\beta}(S, T) X\right\| \\
& \quad \leq\left(\frac{\beta-\alpha}{\alpha}\right)\left\|A_{\alpha}(S, T) X\right\|+\left\|\frac{\beta}{\alpha} A_{\alpha}(S, T) X-A_{\beta}(S, T) X\right\| .
\end{aligned}
$$

From the inequality (5), we have

$$
\left\|\frac{\beta}{\alpha} A_{\alpha}(S, T) X-A_{\beta}(S, T) X\right\| \leq \frac{\beta-\alpha}{\alpha}\left\|A_{\alpha}(S, T) X\right\| .
$$

Thus the right hand side of the inequality (6) is bounded from the above:

$$
\left(\frac{\beta-\alpha}{\alpha}\right)\left\|A_{\alpha}(S, T) X\right\|+\left\|\mid \frac{\beta}{\alpha} A_{\alpha}(S, T) X-A_{\beta}(S, T) X\right\| \leq \frac{2(\beta-\alpha)}{\alpha}\left\|A_{\alpha}(S, T) X\right\| .
$$

Thus we have the inequality (4).

We also have the following proposition. 
Proposition 3.3 Let $S, T, X \in B(\mathcal{H})$ with $S, T \geq 0$. If $0 \leq \alpha<\beta \leq 1$ and $\left\|A_{\beta}(S, T) X\right\|<\infty$, then we have, for any unitarily invariant norm $\|\cdot\| \|$,

$$
\lim _{\alpha^{\prime} \rightarrow \alpha}\left\|A_{\alpha}(S, T) X-A_{\alpha^{\prime}}(S, T) X\right\|=0 .
$$

Proof We firstly prove (7) for the case $0<\alpha<\beta \leq 1$. For $\alpha^{\prime} \in[\alpha, \beta)$, we have

$$
\left\|A_{\alpha}(S, T) X-A_{\alpha^{\prime}}(S, T) X\right\| \leq \frac{2\left(\alpha^{\prime}-\alpha\right)}{\alpha}\left\|A_{\alpha}(S, T) X\right\| \leq \frac{2\left(\alpha^{\prime}-\alpha\right)}{\alpha}\left\|A_{\beta}(S, T) X\right\|
$$

by Proposition 3.2. For $\alpha^{\prime} \in[\alpha / 2, \alpha]$, we similarly have

$$
\left\|A_{\alpha}(S, T) X-A_{\alpha^{\prime}}(S, T) X\right\| \leq \frac{2\left(\alpha-\alpha^{\prime}\right)}{\alpha^{\prime}}\left\|A_{\alpha^{\prime}}(S, T) X\right\| \leq \frac{4\left|\alpha^{\prime}-\alpha\right|}{\alpha}\left\|A_{\beta}(S, T) X\right\| .
$$

We thus obtain for $\alpha^{\prime} \in[\alpha / 2, \beta)$,

$$
\left\|A_{\alpha}(S, T) X-A_{\alpha^{\prime}}(S, T) X\right\| \leq \frac{4\left|\alpha^{\prime}-\alpha\right|}{\alpha}\left\|A_{\beta}(S, T) X\right\|
$$

which implies (7) for the case $0<\alpha<\beta \leq 1$.

We secondly show (7) for the case $\alpha=0$. When $0<\alpha<\beta \leq 1$, we have

$$
\frac{A_{\alpha}\left(e^{2 t}, 1\right)}{A_{\beta}\left(e^{2 t}, 1\right)}=\frac{\alpha}{\beta} \cdot \frac{\sinh (\beta t) \cosh (\alpha t)}{\cosh (\beta t) \sinh (\alpha t)}=\frac{\alpha}{\beta}+\frac{\alpha}{\beta} \cdot \frac{\sinh ((\beta-\alpha) t)}{\cosh (\beta t) \sinh (\alpha t)} .
$$

If we put $B(s, t) \equiv A_{\alpha}(s, t)-(\alpha / \beta) A_{\beta}(s, t)$, then we have

$$
\frac{B\left(e^{2 t}, 1\right)}{A_{\beta}\left(e^{2 t}, 1\right)}=\frac{\alpha}{\beta} \cdot \frac{\sinh ((\beta-\alpha) t)}{\cosh (\beta t) \sinh (\alpha t)},
$$

which is a positive definite function, as shown in (iii) of Proposition 2.3. We also find that

$$
\frac{A_{0}\left(e^{2 t}, 1\right)}{A_{\beta}\left(e^{2 t}, 1\right)}=\frac{1}{\beta t} \cdot \frac{\sinh (\beta t)}{\cosh (\beta t)}
$$

in the limit $\alpha \rightarrow 0$. Then we put the Fourier transforms $\hat{\phi}_{\alpha, \beta}(t)$ and $\hat{\phi}_{0, \beta}(t)$ of two functions $\phi_{\alpha, \beta}(s)$ and $\phi_{0, \beta}(s)$ in the following:

$$
\begin{aligned}
& \int_{-\infty}^{\infty} e^{i s t} \phi_{\alpha, \beta}(s) d s=\hat{\phi}_{\alpha, \beta}(t) \equiv \frac{\alpha}{\beta} \cdot \frac{\sinh ((\beta-\alpha) t)}{\cosh (\beta t) \sinh (\alpha t)}, \\
& \int_{-\infty}^{\infty} e^{i s t} \phi_{0, \beta}(s) d s=\hat{\phi}_{0, \beta}(t) \equiv \frac{1}{\beta t} \cdot \frac{\sinh (\beta t)}{\cosh (\beta t)} .
\end{aligned}
$$

Since we have $B(1,0)=0$ and $A_{0}(1,0)=0$, we have

$$
\begin{aligned}
& A_{\alpha}(S, T) X-\frac{\alpha}{\beta} A_{\beta}(S, T) X=\int_{-\infty}^{\infty}\left(S_{\text {supp } S}\right)^{i s}\left(A_{\beta}(S, T) X\right)\left(T_{\text {supp } T}\right)^{-i s} \phi_{\alpha, \beta}(s) d s, \\
& A_{0}(S, T) X=\int_{-\infty}^{\infty}\left(S_{\text {supp }}\right)^{i s}\left(A_{\beta}(S, T) X\right)\left(T_{\text {supp } T}\right)^{-i s} \phi_{0, \beta}(s) d s
\end{aligned}
$$


from Theorem 3.4 in [5]. Then we have

$$
\left\|A_{\alpha}(S, T) X-A_{0}(S, T) X\right\| \leq\left(\frac{\alpha}{\beta}+\left\|\phi_{\alpha, \beta}-\phi_{0, \beta}\right\|_{1}\right)\left\|A_{\beta}(S, T) X\right\| .
$$

To prove $\lim _{\alpha \rightarrow 0+}\left\|\phi_{\alpha, \beta}-\phi_{0, \beta}\right\|_{1}=0$, we have only to prove $\lim _{\alpha \rightarrow 0+}\left\|\hat{\phi}_{\alpha, \beta}-\hat{\phi}_{0, \beta}\right\|_{2}=0$ thanks to Lemma 5.8 in [5]. Since we have $\int_{-\infty}^{\infty} \phi_{\alpha, \beta}(s) d s=\hat{\phi}_{\alpha, \beta}(0)=(\beta-\alpha) / \beta$, we have $\int_{-\infty}^{\infty} \phi_{0, \beta}(s) d s=\hat{\phi}_{0, \beta}(0)=1$ in the limit $\alpha \rightarrow 0$. From the fact $\sinh x \geq x$ for $x \geq 0$, we also have $0 \leq \hat{\phi}_{\alpha, \beta}(t), \hat{\phi}_{0, \beta}(t) \leq 1 /(\beta|t|)$. Since $\hat{\phi}_{\alpha, \beta}(t)$ and $\hat{\phi}_{0, \beta}(t)$ are positive definite functions, we have $\hat{\phi}_{\alpha, \beta}(t) \leq \hat{\phi}_{\alpha, \beta}(0)=(\beta-\alpha) / \beta \leq 1$ and $\hat{\phi}_{0, \beta}(t) \leq \hat{\phi}_{0, \beta}(0)=1$. (See Chapter 5 in [9] for some basic properties of the positive definite function.) We thus ob$\operatorname{tain} \hat{\phi}_{\alpha, \beta}(t), \hat{\phi}_{0, \beta}(t) \leq \min (1,1 /(\beta|t|))$ for two $L^{2}$-functions $\hat{\phi}_{\alpha, \beta}$ and $\hat{\phi}_{0, \beta}$. We finally obtain $\left|\hat{\phi}_{\alpha, \beta}(t)-\hat{\phi}_{0, \beta}(t)\right|^{2} \leq 4 \min \left(1,1 / \beta t^{2}\right)$. Since $\min \left(1,1 / \beta t^{2}\right)$ is integrable and $\lim _{\alpha \rightarrow 0} \hat{\phi}_{\alpha, \beta}(t)=$ $\hat{\phi}_{0, \beta}(t)$, we obtain $\lim _{\alpha \rightarrow 0+}\left\|\hat{\phi}_{\alpha, \beta}-\hat{\phi}_{0, \beta}\right\|_{2}=0$ by the Lebesgue dominated convergence theorem.

We note that the assumption $\left\|A_{\beta}(S, T) X\right\|<\infty$ for some $\beta \in(0,1]$ is equivalent to $\| S X+$ $X T \|<\infty$, since we have $\left\|A_{\beta}(S, T) X\right\| \leq\|\| A_{1}(S, T) X\|\leq((2-\beta) / \beta)\| A_{\beta}(S, T) X \|$ using the inequality (3).

\section{Conclusion}

We obtained new and tight bounds for the logarithmic mean for unitarily invariant norm. Our results improved the famous inequalities by Hiai and Kosaki [3, 4]. Concluding this paper, we summarize Theorem 2.5 in the familiar form. From the calculations

$$
G_{1 / m_{1}}(s, t)=\frac{1}{m_{1}} \sum_{k=1}^{m_{1}} s^{(2 k-1) / 2 m_{1}} t^{\left(2 m_{1}-(2 k-1)\right) / 2 m_{1}}
$$

and

$$
A_{1 / m_{2}}(s, t)=\frac{1}{m_{2}}\left(\sum_{k=0}^{m_{2}} s^{k / m_{2}} t^{\left(m_{2}-k\right) / m_{2}}-\frac{1}{2}(s+t)\right),
$$

we have

$$
G_{1 / m_{1}}(S, T) X=\frac{1}{m_{1}} \sum_{k=1}^{m_{1}} S^{(2 k-1) / 2 m_{1}} X T^{\left(2 m_{1}-(2 k-1)\right) / 2 m_{1}}
$$

and

$$
A_{1 / m_{2}}(S, T) X=\frac{1}{m_{2}}\left(\sum_{k=0}^{m_{2}} S^{k / m_{2}} X T^{\left(m_{2}-k\right) / m_{2}}-\frac{1}{2}(S X+X T)\right) .
$$

In addition, from the paper [4], we know that

$$
M_{m_{1} /\left(m_{1}+1\right)}(S, T) X=\frac{1}{m_{1}} \sum_{k=1}^{m_{1}} S^{k /\left(m_{1}+1\right)} X T^{\left(m_{1}+1-k\right) /\left(m_{1}+1\right)}
$$


and

$$
M_{m_{2} /\left(m_{2}-1\right)}(S, T) X=\frac{1}{m_{2}} \sum_{k=0}^{m_{2}-1} S^{k /\left(m_{2}-1\right)} X T^{\left(m_{2}-1-k\right) /\left(m_{2}-1\right)} .
$$

Thus Theorem 2.5 can be rewritten as the following inequalities, which are our main result of the present paper:

$$
\begin{aligned}
\frac{1}{m_{1}}\left\|\sum_{k=1}^{m_{1}} S^{k /\left(m_{1}+1\right)} X T^{\left(m_{1}+1-k\right) /\left(m_{1}+1\right)}\right\| & \leq \frac{1}{m_{1}}\left\|\sum_{k=1}^{m_{1}} S^{(2 k-1) / 2 m_{1}} X T^{\left(2 m_{1}-(2 k-1)\right) / 2 m_{1}}\right\| \| \\
& \leq\left\|\int_{0}^{1} S^{\nu} X T^{1-v} d v\right\| \| \\
& \leq \frac{1}{m_{2}}\left\|\sum_{k=0}^{m} S^{k / m_{2}} X T^{\left(m_{2}-k\right) / m_{2}}-\frac{1}{2}(S X+X T)\right\| \| \\
& \leq \frac{1}{m_{2}}\left\|\sum_{k=0}^{m_{2}-1} S^{k /\left(m_{2}-1\right)} X T^{\left(m_{2}-1-k\right) /\left(m_{2}-1\right)}\right\|, \|
\end{aligned}
$$

for $S, T, X \in B(\mathcal{H})$ with $S, T \geq 0, m_{1} \geq 1, m_{2} \geq 2$, and any unitarily invariant norm $\|\cdot\| \cdot \|$.

We have also shown some properties for our means such as monotonicities and norm continuities in the parameter.

\section{Competing interests}

The author declares that he has no competing interests.

\section{Acknowledgements}

I would like to express my deepest gratitude to Professor Fumio Hiai and Professor Hideki Kosaki for giving me valuable comments to improve this manuscript. I also was partially supported by JSPS KAKENHI Grant Number 24540146.

Received: 8 January 2014 Accepted: 4 April 2014 Published: 06 May 2014

\section{References}

1. Zou, L: Matrix versions of the classical Pólya inequality. ScienceAsia 39, 204-207 (2013)

2. Furuichi, S, Yanagi, K: Bounds of the logarithmic mean. J. Inequal. Appl. 2013, 535 (2013)

3. Hiai, F, Kosaki, H: Comparison of various means for operators. J. Funct. Anal. 163, 300-323 (1999)

4. Hiai, F, Kosaki, H: Means for matrices and comparison of their norms. Indiana Univ. Math. J. 48, 899-936 (1999)

5. Hiai, F, Kosaki, H: Means of Hilbert Space Operators. Lecture Notes in Mathematics, vol. 1820. Springer, Berlin (2003)

6. Hiai, F: Matrix analysis: matrix monotone functions, matrix means, and majorization. Interdiscip. Inf. Sci. 16, 139-248 (2010)

7. Kosaki, H: Positive definiteness of functions with applications to operator norm inequalities. Mem. Am. Math. Soc. 212, 997 (2011)

8. Bhatia, R, Kosaki, H: Mean matrices and infinite divisibility. Linear Algebra Appl. 424, 36-54 (2007)

9. Bhatia, R: Positive Definite Matrices. Princeton University Press, Princeton (2007)

10.1186/1029-242X-2014-158

Cite this article as: Furuichi: Unitarily invariant norm inequalities for some means. Journal of Inequalities and Applications 2014, 2014:158 